K. Oyanagi

M. Wada

\section{Neuropathology of parkinsonism-dementia complex and amyotrophic lateral sclerosis of Guam: an update}

\begin{abstract}
A comparative study was performed to investigate the differences and similarities of the neuropathological findings in the parkinsonismdementia complex (PDC) and amyotrophic lateral sclerosis (ALS) of Guam, progressive supranuclear palsy and classic ALS. Based on the findings, it is proposed that (a) PDC is a discrete disease entity, (b) NFTs in Chamorro ALS are merely a background feature widely distributed in this population,
\end{abstract}

(c) Chamorro ALS is a disease combined with classic ALS and neurofibrillary degeneration, (d) thus a subtype of "Guam ALS" is not present, and (e) PDC and ALS of Guam are different diseases.

Key words Parkinsonism-dementia complex $\cdot$ Progressive supranuclear palsy $\cdot$ Amyotrophic lateral sclerosis . Guam · Neuropathology

\section{Introduction}

Since the discovery of high incidence of amyotrophic lateral sclerosis (ALS) and parkinsonism-dementia complex (PDC) in Guam after World War II [2, 16, 18, 23, 50], it was thought Guam ALS was an unique disease diffferent from classic ALS elsewhere [14, 25]. An Unitarian theory that Chamorro PDC and ALS were a single disease entity with different clinical and pathological spectra of expression was proposed by various investigators in the ensuing years $[4,6,10,14,16,17,18,25$, $39,47]$. It was based on the observations of overlapping of neuropathological features in both diseases. To date, the controversy is still going on. In addition to this confusion, vertical gaze palsy was reported in PDC in recent years [3, 24, 45], which has raised another question of the presence of progressive supranuclear palsy (PSP) in the Chamorro population and requires clarification.

The present study included 175 autopsies performed by the author and colleagues on Guam between 1979 and 1982 as a cooperative study with NINCDS, NIH. Those were clinico- pathologically diagnosed as PDC (50 cases), ALS (11 cases), PDC with ALS (PDC-ALS) (5 cases) and others. In addition, two cases autopsied in 1993 were available for the examination.

Based on the findings on these cases and other neurodegenerative diseases in Japan, and the findings reported so far, a comprehensive study was performed to investigate the differences and similarities among PDC and ALS of Guam, PSP and classic ALS.

\section{Neuropathology of the parkinsonism-dementia complex}

\section{Brain atrophy}

The average brain weight of PDC patients was about $1070 \mathrm{~g}$. The cerebrum showed diffuse atrophy accentuated in the frontal and temporal lobes. The thickness of the cerebral cortex 
was generally reduced, especially in the hippocampus and parahippocampal gyrus (Fig. 1a). The basal ganglia and
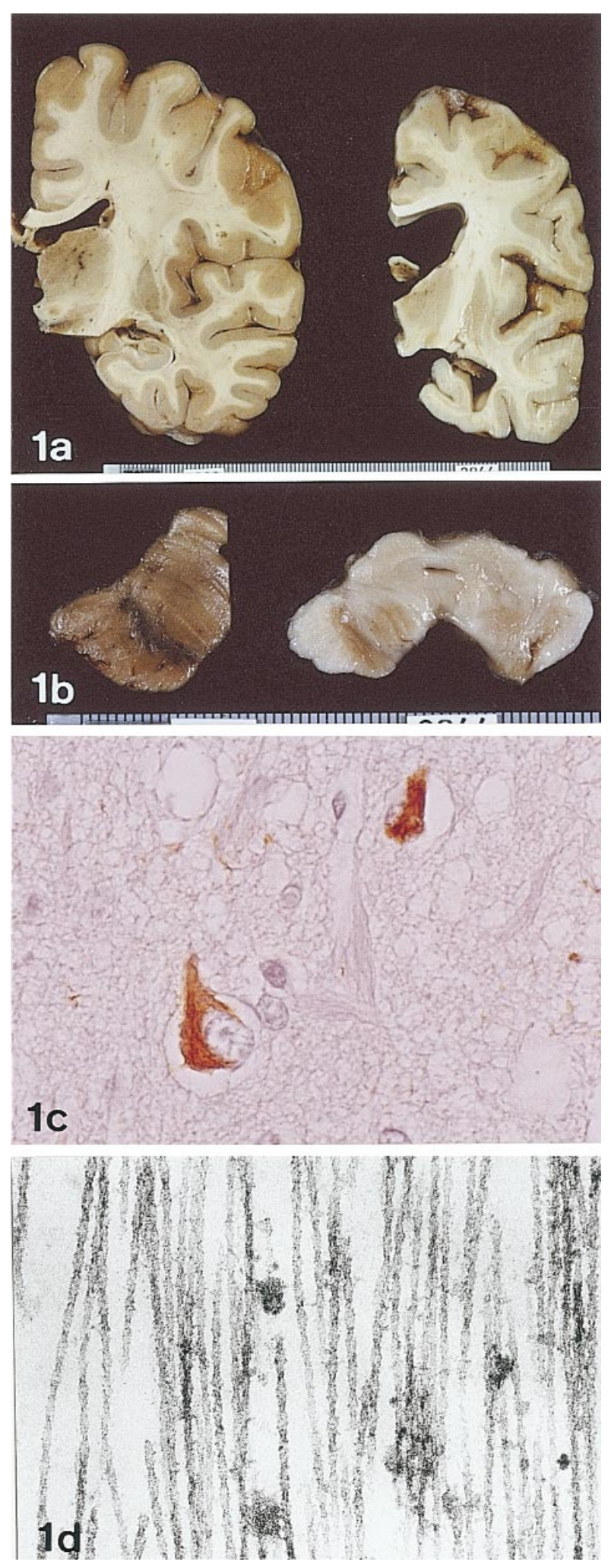

thalamus showed moderate atrophy, and the white matter of the cerebrum and brainstem was diffusely atrophic. The lateral and third ventricles were moderately dilated. The midbrain and pons showed atrophy that paralleled that of the cerebrum. The pigmentation of the substantia nigra and locus ceruleus was competely depleted (Fig. 1b). But the volume of the superior colliculus (Fig. 1b), cerebellum and medulla oblongata was preserved [30, 34].

\section{Cerebral cortex and white matter}

The topographic distribution of brain atrophy was roughly coincided with that of neuronal loss and neurofibrillary tangles (NFTs) [18, 19, 30, 34]. Complete loss of neurons was seen in the Sommer sector, and severe to moderate loss was observed in the temporal, insular and frontal cortices. Many NFTs were observed in the Sommer sector, parahippocampal gyrus, temporal neocortex, and frontal cortex $[18,19,30,33]$. The NFTs were immunopositive for tau (Fig. 1c) [27, 42], $\beta$ [22, 41] and Apo E [5], and mostly composed of paired helical filaments (PHFs) and partly of straight tubules (STs) (Fig. 1d) $[15,30,33]$. Much granulovacuolar degeneration and many Hirano bodies were seen in Ammon's horn [15, 18, 30]. Except in a few cases, senile plaques were only rarely observed in the majority of cases $[18,30]$. Curly fibers/threads were rarely seen (Fig. 1c) [30], and ballooned neurons, Pick bodies or spongiform changes were absent.

The cerebral white matter showed severe atrophy, but myelin pallor was not obvious in most cases [30].

\section{Basal ganglia and thalamus}

The basal nucleus of Meynert showed severe loss of large neurons and many NFTs [18, 26, 28, 32]. The number of large neurons in the neostriatum was decreased to $40 \%$ of that in

Fig. 1 a Coronally cut surface of brains of a patient with parkinsonismdementia complex (PDC) (on the right) and an age-matched non-PDC non-amyotrophic lateral sclerosis (ALS) subject (on the left). b Severe depletion of the pigmentation in the substantia nigra of a patient with PDC (on the right). The size of the superior colliculus looks relatively well preserved. On the left, the midbrain of a non-PDC non-ALS subject. c A number of neurons in the cerebral cortex in PDC show robust immunohistochemical staining for human tau protein. The extracellular ghost tangles were negative for the staining. X510. d Electron micrograph of neurofibrillary tangles (NFTs) in a neuron in the subiculum of a PDC patient. The NFTs are composed mainly of 11 - to 25 -nm-wide paired helical filaments (PHFs) with a periodicity of 63 to $87 \mathrm{~nm}$. Uranyl acetatelead citrate. X55000. 


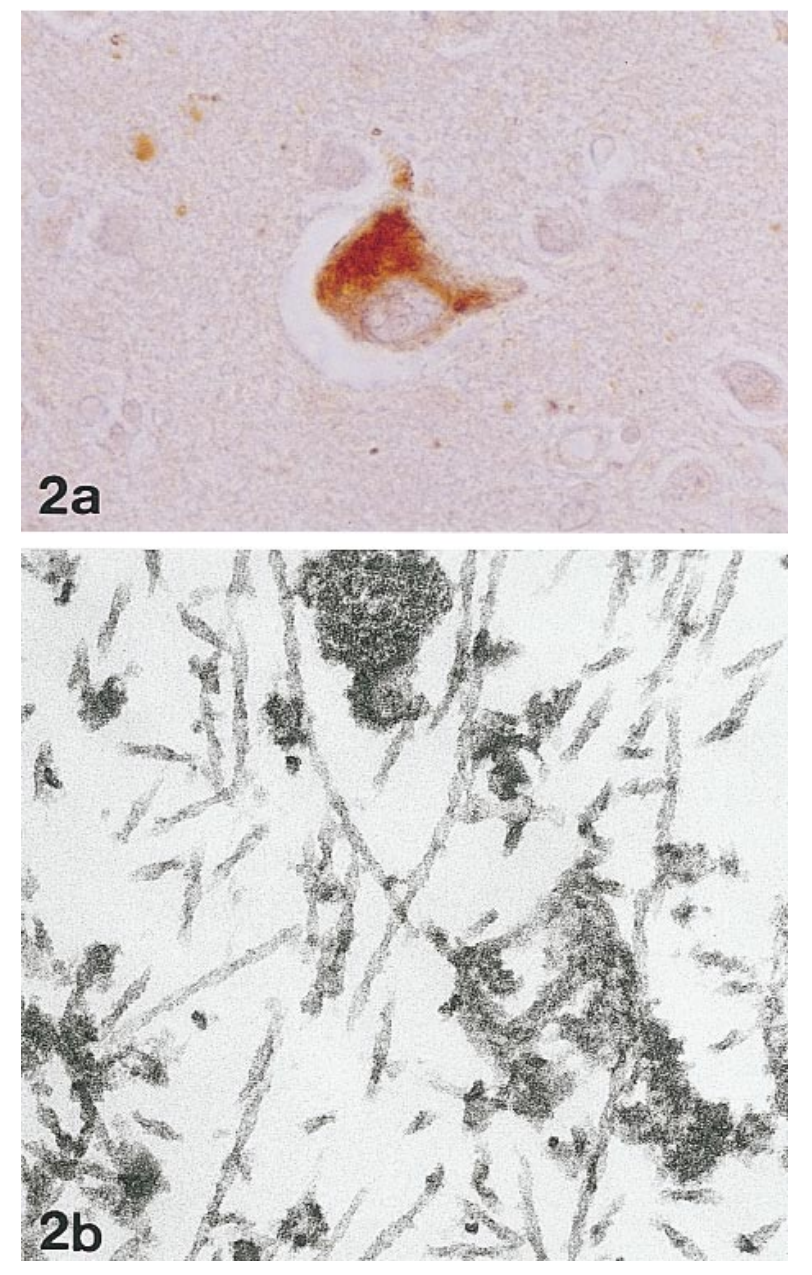

Fig. 2 a A large neuron in the putamen in a patient with PDC. Immunohistochemical staining for human tau protein. X510. b Ultrastructure of NFTs in large neuron of the caudate nucleus in a patient with PDC. The NFTs are composed mainly of PHFs, measuring 21-27 nm in diameter with a periodicity of $65-95 \mathrm{~nm}$. Uranyl acetate-lead citrate. X68000.

controls, and that in the nucleus accumbens to $10 \%$ of the control level [32]. The large neurons in the basal nucleus of Meynert and neostriatum were correspondingly decreased. Remaining large neurons in the neostriatum frequently contained NFTs/tau protein (Fig. 2a) composed of PHFs mainly and STs partly (Fig. 2b) [32]. The globus pallidus showed moderate neuronal loss and NFTs.

The thalamus showed moderate neuronal loss and NFTs in the lateral nucleus and slight loss of neurons with NFTs in the medial nucleus [30]. Severe loss of neurons and many NFTs were evident in the hypothalamus $[18,30]$. The subthalamic nucleus showed slight loss of neurons and a small number of NFTs [30].
Brain stem

Extremely severe neuronal loss was observed in the substantia nigra $[18,30]$ and ventral tegmental area. Lewy bodies were rare. Severe loss of neurons and many NFTs were seen in the locus ceruleus and superior central nucleus $[18,30]$. NFTs in the locus ceruleus were composed of both PHFs and STs [30]. The pedunculopontine and pontine nuclei showed relatively many NFTs with slight neuronal loss. The superior colliculus contained a small number of NFTs, but the number of large neurons in the deeper layer was preserved [30].

\section{Cerebellum}

The numbers of Purkinje and granule cells were preserved. Although a small number of NFTs were observed in the dentate nucleus, no neuronal loss was evident and no grumose degeneration was seen [30].

\section{Spinal cord}

No marked degeneration was observed in the white matter. The anterior horn cells appeared shrunken, but were not reduced in number. A small number of NFTs composed of STs were observed in the intermediate zone and posterior horn, and occasionally in the anterior horn [33].

\section{Glial inclusions}

Tau-immunopositive and Gallyas-positive glial inclusions were observed in the patients with PDC. Granular hazy inclusions were observed in the astrocytes in the amygdaloid nucleus, motor cortex, and inferior olivary nucleus (Fig. 3a, b) [31]. Crescent/coiled inclusions were present in the oligodendroglia of the anterior nucleus of the thalamus, motor cortex, midbrain tegmentum, and the pyramids (Fig. 3c, d) [31]. Tufted astrocytes $[11,49]$ were rarely observed, and astrocytic plaques, which have been considered as a specific finding of corticobasal degeneration (CBD) [11], were not seen.

\section{Neuropathological comparison between PDC and PSP}

\section{Cerebral cortex}

The cerebrum of typical PSP patients was relatively well preserved, and the brain weight was not significantly decreased [43], although severe to moderate loss of neurons 

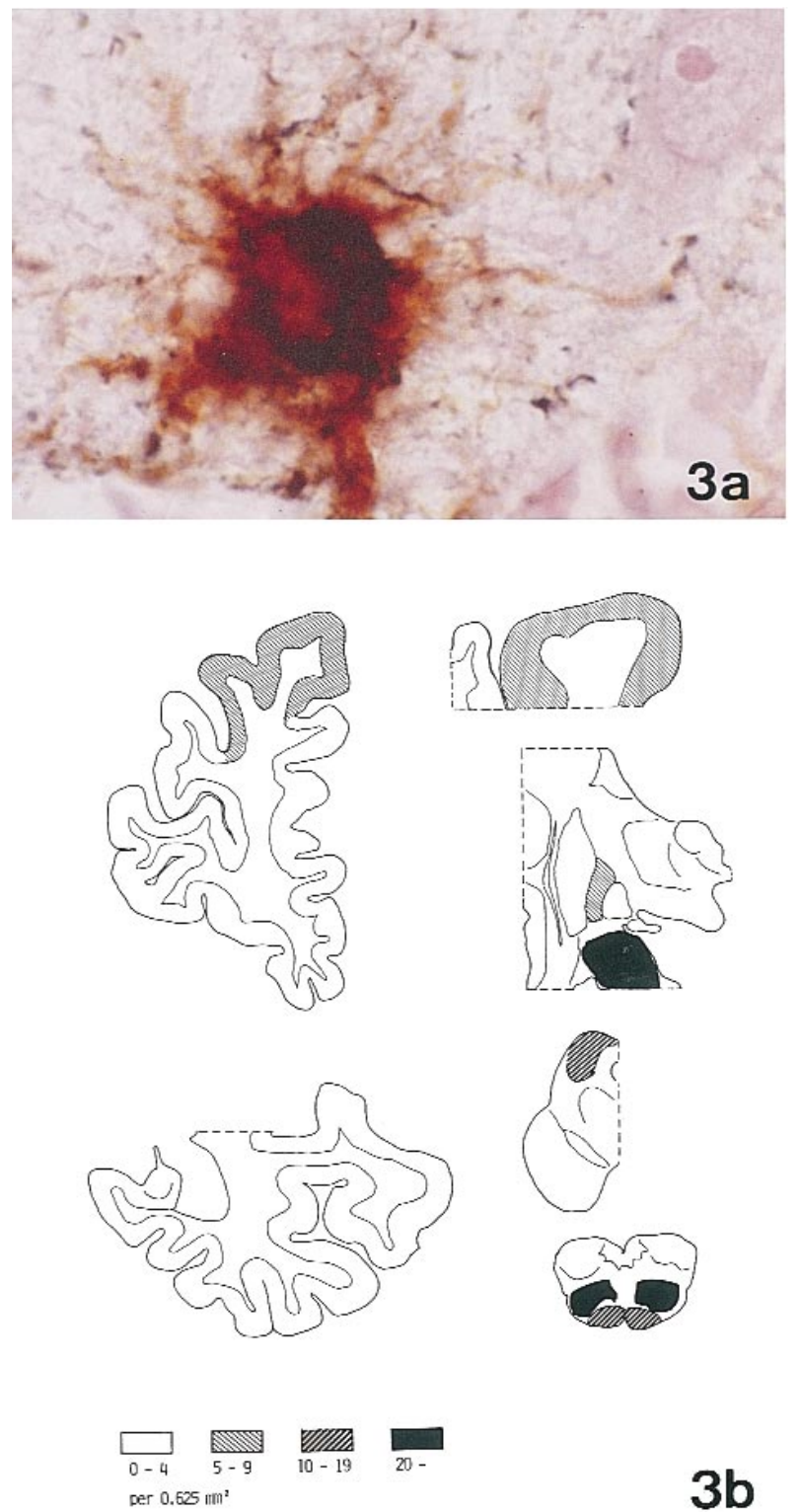

$3 b$

Fig. 3 a Granular hazy inclusion in an astrocytes in the motor cortex of a patient with PDC. Double staining involving Gallyas preparation (black) and glial fibrillary acidic protein immunostaining (brown). X1270. b Schematic demonstration of the topographic distribution of the granular hazy astrocytic inclusions. c Crescent /coiled inclusion in a small round cell in the white matter of the parahipocampal gyrus of a patient with PDC. Gallyas preparation. X1270. d Schematic demonstration of the topographic distribution of the crescent/coiled oligodendroglial inclusions.

was observed in the cerebral cortex in PDC $[18,30]$. It was reported, however, that PSP patients show many NFTs in the cerebral cortex $[13,20]$. These NFTs were located in the superficial layers [20], resembling the appearance of PDC [21]. In
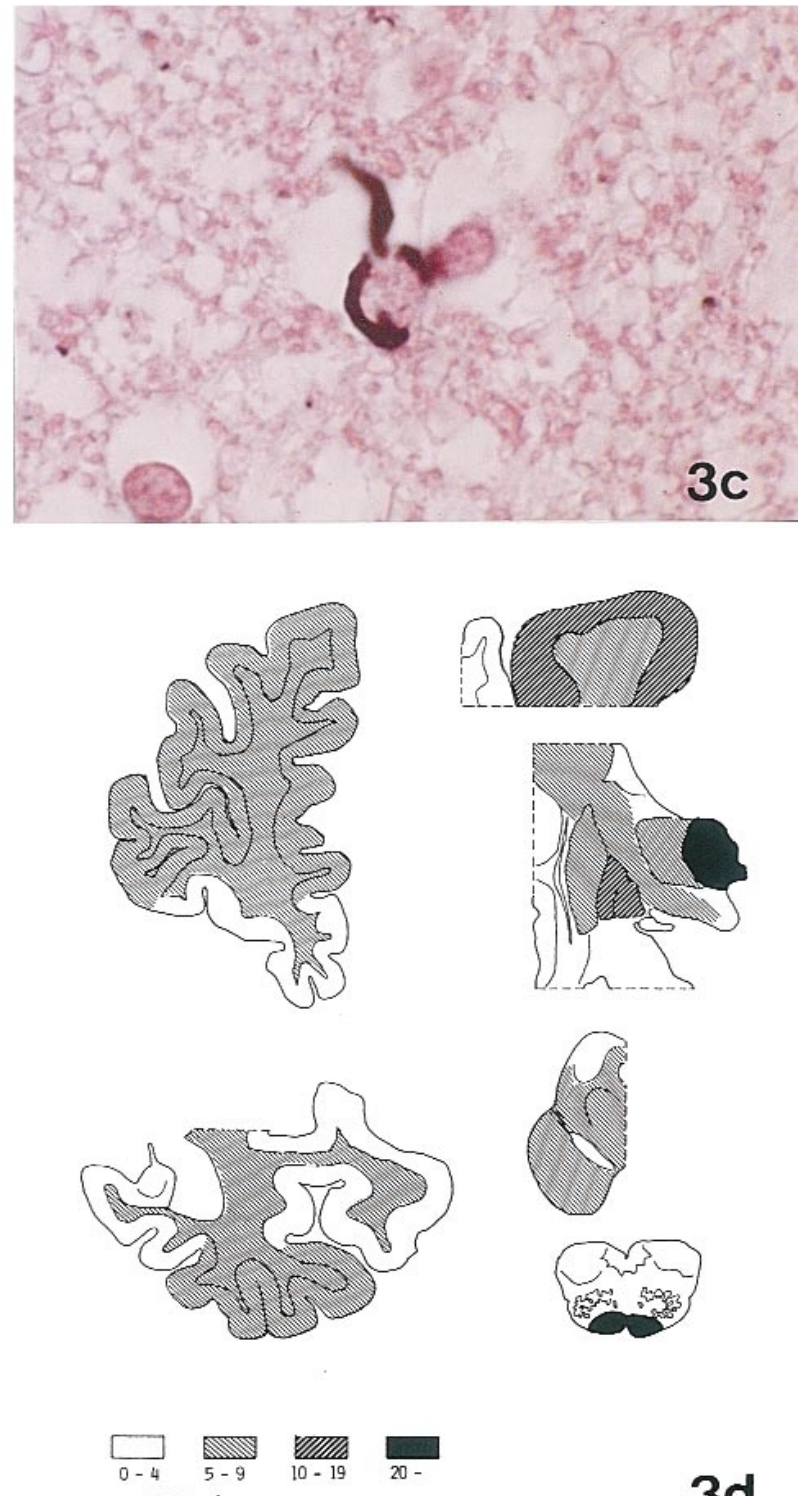

$3 d$

our experience, however, the NFTs in the cerebral neocortex in PSP patients were composed mainly of STs [44], as reported previously in the subcortex [46], whereas PHFs were mainly present in the cerebral cortex in PDC [15, 30].

\section{Basal ganglia}

The large neurons in the neostriatum and the nucleus accumbens in PSP patients decreased diffusely without an accentuation in the nucleus accumbens, and the remaining large neurons frequently contained NFTs. The NFTs were composed mainly of STs and partly PHFs [36]. The number of large neurons in the basal nucleus of Meynert was preserved in PSP 
[35]. Involvement of the subthalamic nucleus was severer in PSP than in PDC [30].

On the other hand, the large neurons in the basal nucleus of Meynert and neostriatum were correspondingly decreased in PDC. Remaining large neurons in the neostriatum frequently contained NFTs composed of PHFs mainly and STs partly in PDC [32].

\section{Brain stem}

Loss of neurons in the substantia nigra in PSP patients was extremely severe both in the pigmented and nonpigmented neurons. The involvement of nonpigmented neurons in the pars reticulata was complete [38], and the finding was different from that of PDC, in which nonpigmented neurons decreased diffusely, but compared with pigmented neurons, they were relatively well preserved in the substantia nigra [38]. Involvement of the superior colliculus in PDC was milder than that of PSP, and the loss of neurons in the ventral tegmental area in PDC was severer than that in PSP [30].

\section{Glial inclusions}

Many tufted astrocytes [11,49] were observed in PSP patients, whereas granular hazy astrocytic inclusion bodies, which were observed in PDC [31], were not evident in PSP; on the other hand, tufted astrocytes were scarcely evident in PDC [31].

\section{Nature of ALS on Guam}

Among 175 autopsy cases, 16 Chamorros were diagnosed clinicopathologically as ALS, and these included 2 cases of classic ALS and 5 cases of combined PDC-ALS. These Chamorro ALS patients clearly showed the pathology of classic ALS, i.e., loss of Betz cells, degeneration of the lateral and anterior corticospinal tracts (Fig. 4a), loss of anterior horn cells and neurons in the hypoglossal nucleus, and presence of Bunina bodies (Fig. 4b) and skein-like inclusions. Relative preservation of Clarke's column, the intermediolateral nucleus and Onuf's nucleus was evident. While the two classic ALS cases that we studied showed a very small number of NFTs, the other 9 ALS patients showed relatively many NFTs and slight degeneration of the substantia nigra, and the 5 combined PDC-ALS cases showed a combination of above-mentioned PDC pathology and ALS pathology [33].

\section{Bunina bodies in Chamorro ALS}

Investigation of the Bunina bodies is inevitably important in ALS research to pursue the pathoetiology and pathomech-
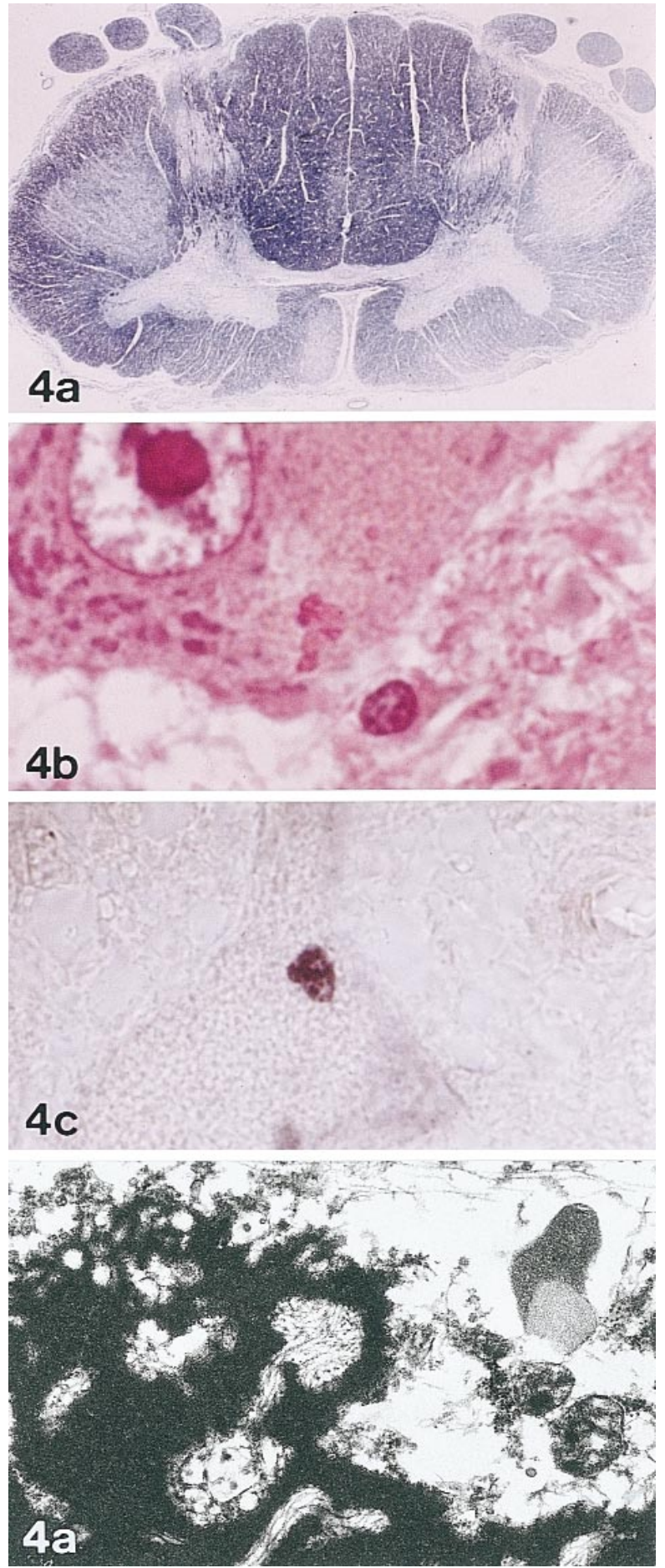

Fig. 4 a Degeneration of the lateral and anterior corticospinal tracts and the anterolateral funiculus, and atrophy of the anterior horn in the cervical spinal cord of a Guamanian ALS patient. Klüver-Barrera staining. b Bunina bodies in an anterior horn cell in the spinal cord in a Guamanian ALS patient. Hematoxylin-eosin. X1270. c Bunina bodies in an anterior horn cell in the spinal cord in a Guamanian ALS patient. Immunohistochemical staining for cystacin-C. X1270. d Electron micrograph of a Bunina body in an anterior horn cell in the spinal cord in a Guamanian ALS patient. Uranyl-lead. X18000. 
anism. It seems to serve as a finding essential to study the pathogenesis of Chamorro ALS, and factor conclusive to the open question whether Chamorro ALS is the same disease as classic ALS or a different disease. In Chamorro ALS, however, not a precise histochemical and immunohistochemical study, nor an intensive ultrastructural examination was reported.

In our study on histochemistry on Bunina bodies, the findings were consistent with classic ALS [48]. The immunohistochemical study showed that cystatin-C antibodies were intensely labeled Bunina bodies in Chamorro ALS (Fig. 4c) [48]. It was found by a topographic study that Bunina bodies were distributed in the spinal anterior horns and Clarke's column in the spinal cord. Ultrastructural features of Bunina bodies were composed of an electron-dense amorphous/ granular material accompanied by vesicular structures and neurofilaments (Fig. 4d) [48]. These results revealed that pathological features of Bunina bodies in Chamorro ALS were quite identical with those of classic ALS and strongly suggested that the similar degenerative process existed in the anterior horn cells in the spinal cord with Chamorro ALS and classic ALS [48].

\section{NFTs as a background feature in Chamorro ALS}

In the central nervous system of Chamorro ALS, NFTs and loss of neurons have been observed extensively, especially in the regions affected in PDC, in addition to the typical neuropathological features of classic ALS [17, 33]. Because the topographic distribution of NFTs, and of the neuronal loss related them, is similar to that in PDC, because patients with combined ALS and PDC have been identified clinically and pathologically, and because ALS as well as PDC patients are sometimes admixed within a family, it has been proposed that Chamorro ALS and PDC are a single disease entity and that Chamorro ALS is a disease different from classic ALS [14, 25]. However, NFTs, which are a basic feature of PDC and Chamorro ALS, have not been investigated in detail or in large series. It is essential for this kind of evaluation to be performed not only in Chamorro ALS and PDC patients, but also in non-PDC non-ALS Chamorro subjects to assess any significant discrepancy from the background distribution in the population. We therefore conducted a detailed quantitative investigation of NFTs in non-PDC non-ALS Chamorro subjects, and also Chamorro PDC, ALS and PDC-ALS patients.

The topographic distribution of NFTs was basically the same in each disease, and also in the non-PDC non-ALS group. There were relatively few, if any, NFTs in non-PDC non-ALS subjects and ALS patients, but in PDC and PDCALS patients there were many, especially in the frontal and temporal neocortex [33]. NFTs were found in the Sommer sector and parahippocampal gyrus in all of non-PDC non-
ALS, PDC, ALS, and PDC-ALS, without any evident difference among them. In the gyrus occipitalis temporalis lateralis, however, the number of Bodian-stained NFTs seemed to be distinct in every group. Non-PDC non-ALS and ALS had less than 123 NFTs, and PDC and PDC-ALS had more than 194 in this region. In the gyrus frontalis superior, non-PDC non-ALS and ALS had less than 12 NFTs, whereas PDC and PDC-ALS showed more than 56 [33].

Thus the studies on so-called Chamorro "control cases" demonstrated that NFTs are the universal neuropathology of aging Chamorro brains. The NFTs in Chamorro ALS are merely a background feature widely distributed in this population $[1,4,9]$. These findings indicated (1) classic ALS does exist on Guam, (2) the mechanism of motor neuron degeneration in Chamorro ALS is basically different from that in PDC, and (3) the cases of combined PDC-ALS are supposed to suffer from two distinct diseases of the central nervous system. The authors think

$$
\begin{aligned}
& \text { Guam ALS }=\text { Classic ALS + NFTs } ? \\
& \text { Guam ALS } \neq \text { PDC } ?
\end{aligned}
$$

The finding that ALS is disappearing while PDC is still prevalent on Guam $[7,12,29,40]$ may support the consideration.

\section{Newly appering neurodegenerative disease on Guam}

Parkinsonism, dementia and vertical gaze palsy in a

Guamanian with atypical neuroglial degeneration

In addition to rigidity, tremor, bradykinesia and dementia, ocular signs have been observed in patients with PDC $[8,16]$. With respect to the characteristics of eye movements in PDC, intact ocular motility (except for impairment of convergence) [16], diminished spontaneous eye blink [8], upward vertical gaze palsy (although this occurs rarely) [8], and supranuclear disturbance of ocular motility [24] have been reported. On the other hand, neuropathological findings that are typical of PDC have even been reported to occur in patients with PSP-like syndrome [3].

A 58-year-old Chamorro female patient, who died in 1993, was examined clinicopathologically. At the age of 51, she suffered from hemiparkinsonism, then bradykinesia, rigidity without tremor, and dementia. Extrapyramidal symptoms developed, and at the age of 57, vertical gaze palsy was noted. The clinical diagnosis was PDC with vertical gaze palsy. The brain showed atrophy in the frontal and temporal lobes, and the atrophy was accentuated in the dentate gyrus, Ammon's horn and parahippocampal gyrus. The substantia nigra and locus ceruleus were completely depigmented. Numerous NFTs were seen in the subiculum and amygdaloid nucleus. Many NFTs 
were evident in the parahippocampal gyrus, lateral occipitotemporal gyrus, insula, Sommer sector, basal nucleus of Meynert, lateral nucleus of the thalamus, subthalamic nucleus and brain stem. The Sommer sector, substantia nigra, locus ceruleus and basal nucleus of Meynert showed severe loss of neurons. These findings were apparently consistent with those associated with PDC. However, in this patient, severe neuronal loss was seen in the subthalamic nucleus and lateral nucleus of the thalamus, and grumose degeneration, which has not previously been reported in PDC, was seen in the dentate nucleus. In addition, many tufted astrocytes, which have been reported to occur in PSP [11] and postencephalitic parkinsonism, but scarcely observed in PDC, were present. Furthermore, astrocytic plaques, which have been considered as a specific finding of CBD [11], were observed in the cerebral cortex. On the other hand, granular hazy astrocytic inclusions, previously reported to occur in PDC [31], were not seen. Chromatolytic neurons were not observed. The question thus arises as to whether it is appropriate to consider this patient as having suffered from a combination of PDC + PSP + CBD. From the view points of absence of granular hazy astrocytic inclusions and chromatolytic neurons, and of tufted astrocytes in the neostriatum, it is conceivable that this patient is a case of a new disease entity [37].

Considering that the clinical diagnosis of the present patient was PDC, study of Guamanians ought to be made based on the precise neuropathological examination.

\section{Conclusions}

PDC is a discrete disease entity,

- NFTs in Chamorro ALS are merely a background feature widely distributed in this population,

$\checkmark$ thus Chamorro ALS is a disease combined with classic ALS and neurofibrillary degeneration,

a subtype of "Guam ALS" is not present, and

- PDC and ALS of Guam are different diseases.

Acknowledgments The authors are indebted to Dr. K.-M. Chen of Guam Memorial Hospital, Guam, U.S.A., Professor Emeritus F. Ikuta of Niigata University, Niigata, Japan, Professor A. Hirano of Montefiore Medical Center, New York, U.S.A., Dr. T. Makifuchi of Saigata National Hospital, Niigata, Japan, Dr. T. Ohtoh of National Kohriyama Hospital, Fukushima, Japan, Professor D. P. Perl of Mt. Sinai School of Medicine, New York, U.S.A., Dr. U. K. Craig of University of Guam, Guam, U.S.A., Dr. T. N. Chase and Dr. D. C. Gajdusek of NINDS, NIH, Bethesda, U.S.A., Dr. R. T. Yanagihara of University of Hawaii, Hawaii, U.S.A., Dr. R. M. Garruto of Binghamton University, New York, U.S.A., and Dr. H.-Y. Park and Dr. A. J. Loerzel of Guam Memorial Hospital, Guam, U.S.A., for their help and encouragement to promote the research. Supported in part by a Grant-in-Aid for Scientific Research (C) 10680714 from the Ministry of Education, Science, Sports and Culture, Japan.

\section{References}

1. Anderson FH, Richardson EP Jr, Okazaki H, Brody JA (1979) Neurofibrillary degeneration on Guam. Frequency in Chamorros and non Chamorros with no known neurological disease. Brain 102: 65-77

2. Arnold A, Edgren DC, Palladino VS (1953) ALS: 50 cases observed on Guam. J Nerv Mental Dis 117: 135-139

3. Bergeron C, Steele JC (1990) Guam parkinsonism-dementia complex and amyotrophic lateral sclerosis. A clinicopathological study of seven cases. In: Rose FC, Norris FH (eds) Amyotrophic Lateral Sclerosis. New Advances in Toxicology and Epidemiology. Smith-Gordon, London, pp 89-98

4. Brody JA, Hirano A, Scott M (1971) Recent neuropathologic observations in ALS and PDC of Guam. Neurology 21: 528-536
5. Buée L, Pérez-Tur J, Leveugle B, BuéeScherer V, Mufson EJ, Loerzel AJ, Chartier-Harlin M-C, Perl DP, Delacourte A, Hof PR (1996) Apolipoprotein E in Guamanian amyotrophic lateral sclerosis/ parkinsonism-dementia complex: genotype analysis and relationship to neuropathological changes. Acta Neuropathol 91: 247253

6. Chen KM (1976) Motor neuron involvement in parkinsonism-dementia and its relationship to Guam ALS. In: Tsubaki T, Toyokura Y (eds) Amyotrophic Lateral Sclerosis. University of Tokyo Press, Tokyo, pp 319-344

7. Chen KM (1995) Disappearance of ALS from Guam: implications for exogenous causes. Clin Neurol 35: 1549-1553

8. Chen K-M, Chase TN (1985) Parkinsonism-dementia. In: Vinken PJ, Bruyn GW, Klawans HL (eds) Handbook of Clinical Neurology, Vol. 49, Revised series 5, Chap 8. Elsevier, Amsterdam, pp 167-183

9. Chen L (1981) Neurofibrillary change on Guam. Arch Neurol 38: 16-18
10. Elizan TS, Hirano A, Abrams BM, Need RL, van Nuis C, Kurland LT (1966) Amyotrophic lateral sclerosis and parkinsonismdementia complex of Guam. Arch Neurol 14: 356-368

11. Feany MB, Mattiace LA, Dickson DW (1996) Neuropathologic overlap of progressive supranuclear palsy, Pick's disease and corticobasal degeneration. J Neuropathol Exp Neurol 55: 53-67

12. Garruto RM, Yanagihara R, Gajdusek DC (1985) Disappearance of high-incidence amyotrophic lateral sclerosis and parkinsonism-dementia on Guam. Neurology 35: 193-198

13. Hauw J-J, Verny M, Delaére P, Cervera $P$, He Y, Duyckaerts C (1990) Constant neurofibrillary changes in the neocortex in progressive supranuclear palsy. Basic differences with Alzheimer's disease and aging. Neurosci Lett 119: 182-186 
14. Hirano A, Arumugasamy N, Zimmerman HM (1967) Amyotrophic lateral sclerosis. A comparison of Guam and classical cases. Arch Neurol 16: 357-363

15. Hirano A, Dembizer HM, Kurland LT, Zimmermann HM (1968) The fine structure of some intraganglionic alterations. Neurofibrillary tangle, granulovacuolar bodies and "rod-like" structures as seen in Guam amyotrophic lateral sclerosis and parkinsonism-dementia complex. J Neuropathol Exp Neurol 27: 167-182

16. Hirano A, Kurland LT, Krooth RS, Lessell S (1961) Parkinsonism-dementia complex, an endemic disease on the island of Guam. I. Clinical features. Brain 84: 642-661

17. Hirano A, Malamud N, Elizan TS, Kurland LT (1966) Amyotrophic lateral sclerosis and parkinsonism-dementia complex on Guam. Further pathologic studies. Arch Neurol 15: 35-51

18. Hirano A, Malamud N, Kurland LT (1961) Parkinsonism-dementia complex, an endemic disease on the island of Guam. II. Pathological features. Brain 84: 662-679

19. Hirano A, Zimmerman HM (1962) Alzheimer's neurofibrillary changes. A topographic study. Arch Neurol 7: 227-242

20. Hof PR, Delacourte A, Bouras C (1992) Distribution of cortical neurofibrillary tangles in progressive supranuclear palsy; a quantitative analysis of six cases. Acta Neuropathol 84: 45-51

21. Hof PR, Perl DP, Loerzel AJ, Morrison JH (1991) Neurofibrillary tangle distribution in the cerebral cortex of parkinsonismdementia cases from Guam: differences with Alzheimer's disease. Brain Res 564: 306-313

22. Ito H, Hirano A, Yen S-H, Kato S (1991) Demonstration of $\beta$ amyloid protein-containing neurofibrillary tangles in parkinsonism-dementia complex on Guam. Neuropathol Appl Neurobiol 17: 365-373

23. Koerner DR (1952) ALS on Guam. A clinical study and review of the literature. Ann Int Med 37: 1204-1220

24. Lepore FE, Steele JC, Cox TA, Tillson G, Calne DB, Duvoisin RC, Lavine L, McDarby JV (1988) Supranuclear disturbances of ocular motility in Lytico-Bodig. Neurology 38: 1849-1853

25. Malamud N, Hirano A, Kurland LT (1961) Pathoanatomic changes in amyotrophic lateral sclerosis on Guam. Special reference to the occurrence of neurofibrillary changes. Arch Neurol 5: 401-415
26. Masullo C, Pocchiari M, Marotti P, Macchi G, Garruto RM, Gibbs Jr CJ, Yanagihara R, Gajdusek DC (1989) The nucleus basalis of Leynert in parkinsonism-dementia of Guam: a morphometric study. Neuropathol Appl Neurobiol 15: 193-206

27. Mawal-Dewan M, Schmidt ML, Balin B, Perl DP, Lee VM-Y, Trojanowski JQ (1996) Identification of phosphorylation sites in PHF-tau from patients with Guam amyotrophic lateral sclerosis/parkinsonismdementia complex. J Neuropathol Exp Neurol 55: 1051-1059

28. Nakano I, Hirano A (1983) Neuron loss in the nucleus basalis of Meynert in parkinsonism-dementia complex of Guam. Ann Neurol 13: 87-91

29. Okumura H, Chen K-M, Kurland LT (1995) Recent epidemiologic study of amyotrophic lateral sclerosis (ALS) and parkinsonism-dementia complex (PDC) in Guam island. Jpn J Clin Ecol 4: 24-28

30. Oyanagi K, Ikuta F, Chen K-M (1992) Neuropathological features of the parkinsonism-dementia complex of Guam. In: Yanagisawa K, Miyatake T (eds) Dementia. Persuit of Its Causative Lesions. Kagakuhyoronsha, Tokyo, pp 36-56

31. Oyanagi K, Makifuchi T, Ohtoh T, Chen K-M, Gajdusek DC, Chase TN (1997) Distinct pathological features of the Gallyasand tau-positive glia in the parkinsonismdementia complex and amyotrophic lateral sclerosis of Guam. J Neuropathol Exp Neurol 56: 308-316

32. Oyanagi K, Makifuchi T, Ohtoh T, Chen K-M, Gajdusek DC, Chase TN, Ikuta F (1994) The neostriatum and nucleus accumbens in parkinsonism-dementia complex of Guam: a pathological comparison with Alzheimer's disease and progressive supranuclear palsy. Acta Neuropathol 88: $122-128$

33. Oyanagi K, Makifuchi T, Ohtoh T, Chen KM, van der Schaaf T, Gajdusek DC, Chase TN, Ikuta F (1994) Amyotrophic lateral sclerosis of Guam: the nature of the neuropathological findings. Acta Neuropathol 88: 405-412

34. Oyanagi K, Makifuchi T, Ohtoh T, Ikuta F, Chen K-M, Chase TN, Gajdusek DC (1994) Topographic investigation of brain atrophy in parkinsonism-dementia complex of Guam: a comparison with Alzheimer's disease and progressive supranuclear palsy. Neurodegeneration 3: 301-304

35. Oyanagi K, Takahashi H, Wakabayashi K, Ikuta F (1989) Correlative decrease of large neurons in the neostriatum and basal nucleus of Meynert in Alzheimer's disease. Brain Res 504: 354-357
36. Oyanagi K, Takahashi H, Wakabayashi K, Ikuta F (1991) Large neurons in the neostriatum in Alzheimer's disease and progressive supranuclear palsy: a topographic, histographic and ultrastructural investigation. Brain Res 544: 221-226

37. Oyanagi K, Chen K-M, Craig UK, Yamazaki M, Perl PP (in press) Parkinsonism, dementia and vertical gaze palsy in a Guamanian with atypical neuroglial degeneration. Acta Neuropathol

38. Oyanagi $\mathrm{K}$ et al. Unpublished data

39. Perl DP (1998) ALS-PDC of Guam. In: Markesbery W (ed) The Neuropathology of Dementing Disorders. W. Arnold, London, pp 68-92

40. Reed D, Labarthe D, Chen KM, Stallones R (1987) A cohort study of amyotrophic lateral sclerosis and parkinsonism-dementia on Guam and Rota. Am J Epidemiol 125: 92-100

41. Schmidt ML, Lee VM-Y, Saido T, Perl D, Schuck T, Iwatsubo T, Trojanowski JQ (1998) Amyloid plaques in Guam amyotrophic lateral sclerosis/parkinsonismdementia complex contain species of $\mathrm{A} \beta$ similar to those found in the amyloid plaques of Alzheimer's disease and pathological aging. Acta Neuropathol 95: 117122

42. Shankar SK, Yanagihara R, Garruto RM, Grundke-Iqbal I, Kosik KS, Gajdusek DC (1989) Immunocytochemical characterization of neurofibrillary tangles in amyotrophic lateral sclerosis and parkinsonismdementia of Guam. Ann Neurol 25: 146151

43. Steele J, Richardson JC, Olszewski J (1964) Progressive supranuclear palsy. A heterogeneous degeneration involving the brain stem, basal ganglia and cerebellum with vertical gaze and pseudobulbar palsy, nuchal dystonia and dementia. Arch Neurol 10: $333-359$

44. Takahashi H, Oyanagi K, Takeda S, Hinokuma K, Ikuta F (1989) Occurrence of 15 -nm-wide straight tubules in neocortical neurons in progressive supranuclear palsy. Acta Neuropathol 79: 233-239

45. Tanner CM, Steele JC, Perl DP, Schoenberg BS (1987) Parkinsonism, dementia, and gaze paresis in Chamorros on Guam: a progressive supranuclear palsy-like syndrome. Ann Neurol 22: 174 
46. Tellez-Nagel I, Wisniewski HM (1973) Ultrastructure of neurofibrillary tangles in Steele-Richardson-Olszewski syndrome. Arch Neurol 29: 324-327
47. Ueda T (1993) Possible linkage between ALS ans PDC on Guam: the nosological significance of overlapped cases. Wakayama Med Report 34: 35-51

48. Wada M, Uchihara T, Nakamura A, Oyanagi K (1999) Bunina bodies in amyotrophic lateral sclerosis on Guam: a histochemical, immunohistochemical, and ultrastructural investigation. Acta Neuropathol 98: 150-156
49. Yamada T, McGeer PL, McGeer EG (1992) Appearance of paired nucleated, Taupositive glia in patients with progressive supranuclear palsy brain tissue. Neurosci Lett 135: 99-102

50. Zimmerman HM (1945) Monthly report to the medical officer in command. US Naval Medical Research Unit 2. Guam. June 1 